\title{
Role of Thr199 residue in human $\beta$-Carbonic anhydrase-II pH-dependent activity elucidated by microsecond simulation analysis
}

\author{
Pulala Raghuveer Yadav ${ }^{1 *}$, Syed Hussain Basha ${ }^{2}$, Pavan Kumar Pindi ${ }^{3 *}$
}

1. Department of Biotechnology, Indian Institute of Technology Hyderabad, Kandi, Sangareddy 502285, Telangana, India.

2. Innovative Informatica Technologies, Hyderabad 500049, Telangana, India.

3. Department of Microbiology, Palamuru University, Mahabubnagar 509001, Telangana, India.

\section{Correspondence to be addressed:}

Email:pr.yadav@bt.iith.ac.in or pavankumarpindi@gmail.com

\begin{abstract}
Carbonic anhydrases catalyze the reversible hydration of carbon dioxide to form bicarbonate, a reaction required for many functions such as carbon assimilation, $\mathrm{pH}$ acid-base homeostasis, respiration and photosynthesis via zinc-hydroxide mechanism for carbon dioxide hydration. In earlier studies, it was revealed that Carbonic anhydrases are inactive at $\mathrm{pH} 7.5$ and active at $\mathrm{pH}$ 8.4. This steep $\mathrm{pH}$ dependence for its activity led us to design this work in order to understand its mode of action at atomic level detailing. In our microsecond simulation based analysis, it was revealed that the interaction between Glu106 and Thr199 plays a critically important role in its activity. Thr199 co-ordinated loop movement was observed to be acting as a lid, with 'open' and 'close' mechanism for substrate entry to the core of the catalytic site, where $\mathrm{Zn}$-ion resides and executes its carbon dioxide hydration mechanism. On the other hand, decline in the total secondary structural elements percentage in the protein was observed in correspondence to the $\mathrm{pH}$ condition change. Especially, $\alpha$-helices between Thr125-Gly145 and Val150-Lys 170 residues were noticed to be losing their structural integrity responsible for formation of dimer and tetramers. In conclusion, our analysis showed that the interaction between Glu106 and Thr199 is crucial for maintaining the structural integrity of the Thr199 corodinated loop, responsible for allowing substrate towards catalytic site.
\end{abstract}

Keywords: human $\beta$-Carbonic anhydrase-II, Glu106 and Thr199 interaction, carbon dioxide hydration, molecular dynamic simulations, $\mathrm{pH}$ dependent activity.

\section{Introduction:}

Carbonic anhydrase (CA, EC 4.2.1.1), a zinc metalloenzyme, catalyzes the reversible hydration of $\mathrm{CO}_{2}$ to $\mathrm{HCO}_{3}^{-}$[1]. It is involved in processes connected with acid-base homeostasis, photosynthesis and respiration [2]. HCAII enzyme is a functional 29-kDa monomer consisting of a 10-stranded, twisted beta-sheet. There are five CA families $(\alpha, \beta, \gamma, \delta$, and $\zeta)$. Their amino acid sequences have no significant similarity, that is, they are evolutionarily independent [3]. The active site is located at the bottom of a $15-\AA ̊$ cone-shaped cavity that leads to the center of the protein. Key features of the active site include a zinc ion coordinated tetrahedrally by 3 histidine residues His-94, His-96, and His-119 [4]. 
On the other hand, the accumulation of carbon dioxide (CO2) is the main cause of global climate change and it is very important to develop technologies that can reduce atmospheric $\mathrm{CO} 2$ level [5]. Although several $\mathrm{CO}_{2}$ capture processes are under development [6], CA has been considered as an important biocatalyst due to its highest efficiency of $\mathrm{CO} 2$ conversion via hydration for $\mathrm{CO} 2$ capture system development [7]. The generally accepted catalytic mechanism of CA is described by a 3-step kinetic scheme: (i) a $\mathrm{Zn}-\mathrm{OH}_{-}$moiety catalyzes the inter-conversion of carbon dioxide to bicarbonate, leaving a $\mathrm{H}_{2} \mathrm{O}$ molecule as the fourth zinc ligand (ii) a proton from the zinc-bound $\mathrm{H}_{2} \mathrm{O}$ is then transferred to the His-64 imidazole ring and (iii) this proton then leaves His-64 for the surrounding solvent [8]. In earlier studies, it was revealed that Carbonic anhydrases are inactive at $\mathrm{pH} 7.5$ and active at $\mathrm{pH}$ 8.4. This steep $\mathrm{pH}$ dependence for its activity led us to design this work in order to understand its mode of action at atomic level detailing.

\section{Methods:}

Schrodinger's maestro visualization program v9.6 [9] was utilized to visualize the protein, hydrogen bonding network, to calculate length of the bonds and to render images. Crystal structure of Carbonic Anhydrase - II (CA-II) [PDB: 2VVB] was imported from the Protein Data Bank (PDB) [10]. Schrodinger's Desmond module [11] was used for molecular dynamic simulation studies. OPLS 2005 force field [12] parameters have been applied to simulation TIP3P water models [13]. Periodic boundary conditions were used to determine the specific size and shape of the water box buffered at $10 \AA$ distances and box volume was calculated as $\sim 300000$ cubic Ås of simulation box volume respectively. Before starting the analysis, we have made sure that all the simulations were carried out at same temperature, pressure and volume conditions throughout the simulated timescale. As part of the simulation quality analysis, it was revealed that average total energy of the simulated system remains the same as approximately $7500 \mathrm{kcal} / \mathrm{mol}$ in both cases of simulations (figure 1 ).

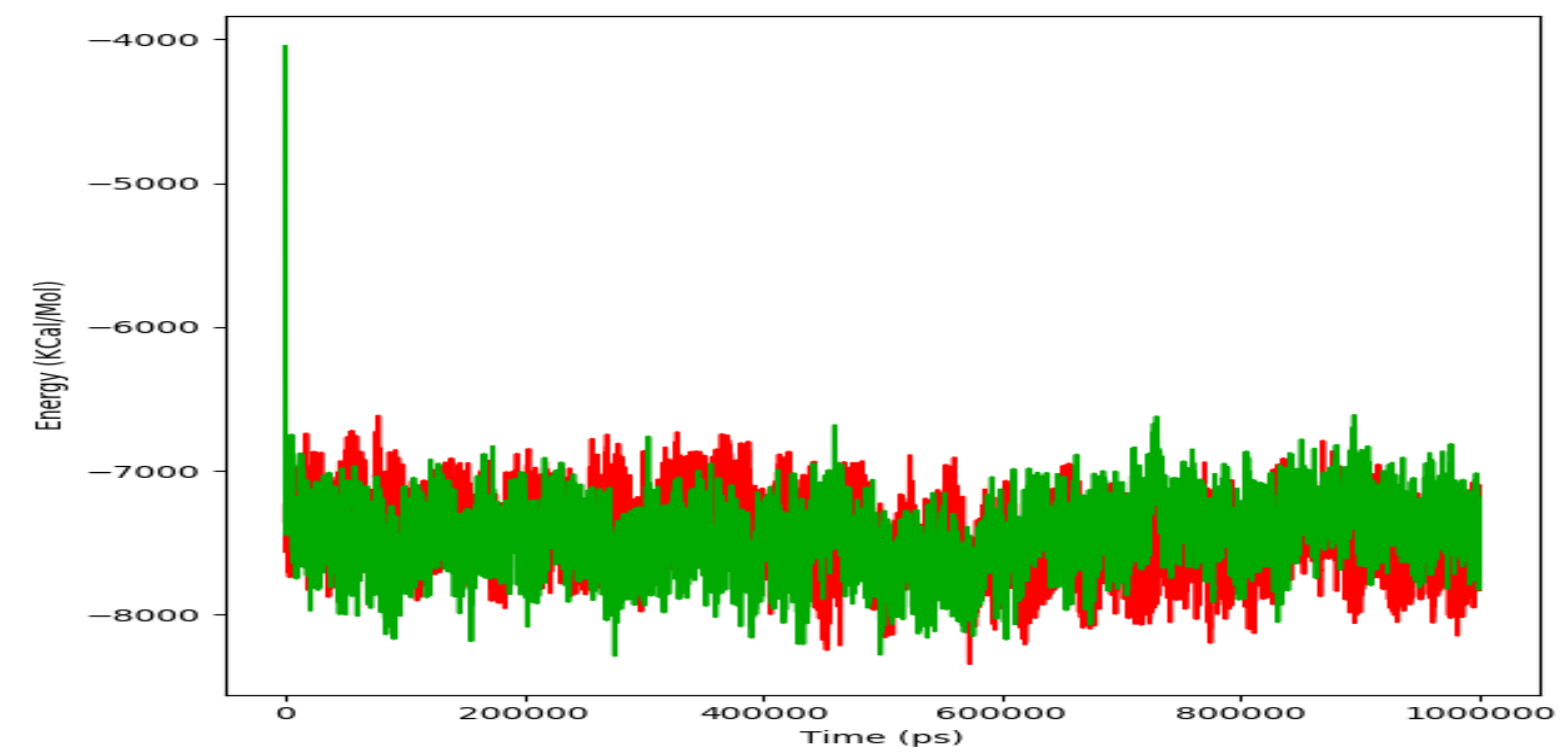

Figure 1: Energy analysis graphs of the inactive (red) at $\mathrm{pH} 7.5$ and active (green) at $\mathrm{pH} 8.4 \mathrm{CA}-\mathrm{II}$ protein. 


\section{Results \& Discussion:}

In order to understand the dynamics of the CA-II in its inactive and active forms, we have performed two different molecular dynamic simulation of 1 microsecond (1000 nanoseconds) each. In one simulation, we have simulated CA-II in its apo form at $\mathrm{pH} \mathrm{7.5,} \mathrm{where} \mathrm{it} \mathrm{was}$

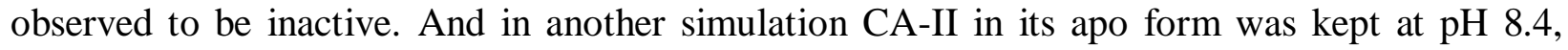
where it was reported to be active [14].

\section{Analysis of CA-II dynamics during the simulated timescale:}

In order to understand the CA-II dynamics, we have analyzed Root mean square deviation (RMSD) of protein backbone, Radius of Gyration (ROG) of protein, Root mean square fluctuations (RMSF) of individual residues in the protein, total number of intra molecular hydrogen bonds found within the protein and Secondary structural elements (SSE) percentage with reference to the simulated timescale. Protein's backbone RMSD of CA-II (figure 2) was observed to be fluctuating between 1.5 and $3.0 \AA$, with an average of 2.2 and $2.4 \AA$ in its inactive and active form respectively. Calculated RMSD observed via superimposition of CA-II protein backbone at first and last frames from above mentioned simulations are shown in figure 3 .

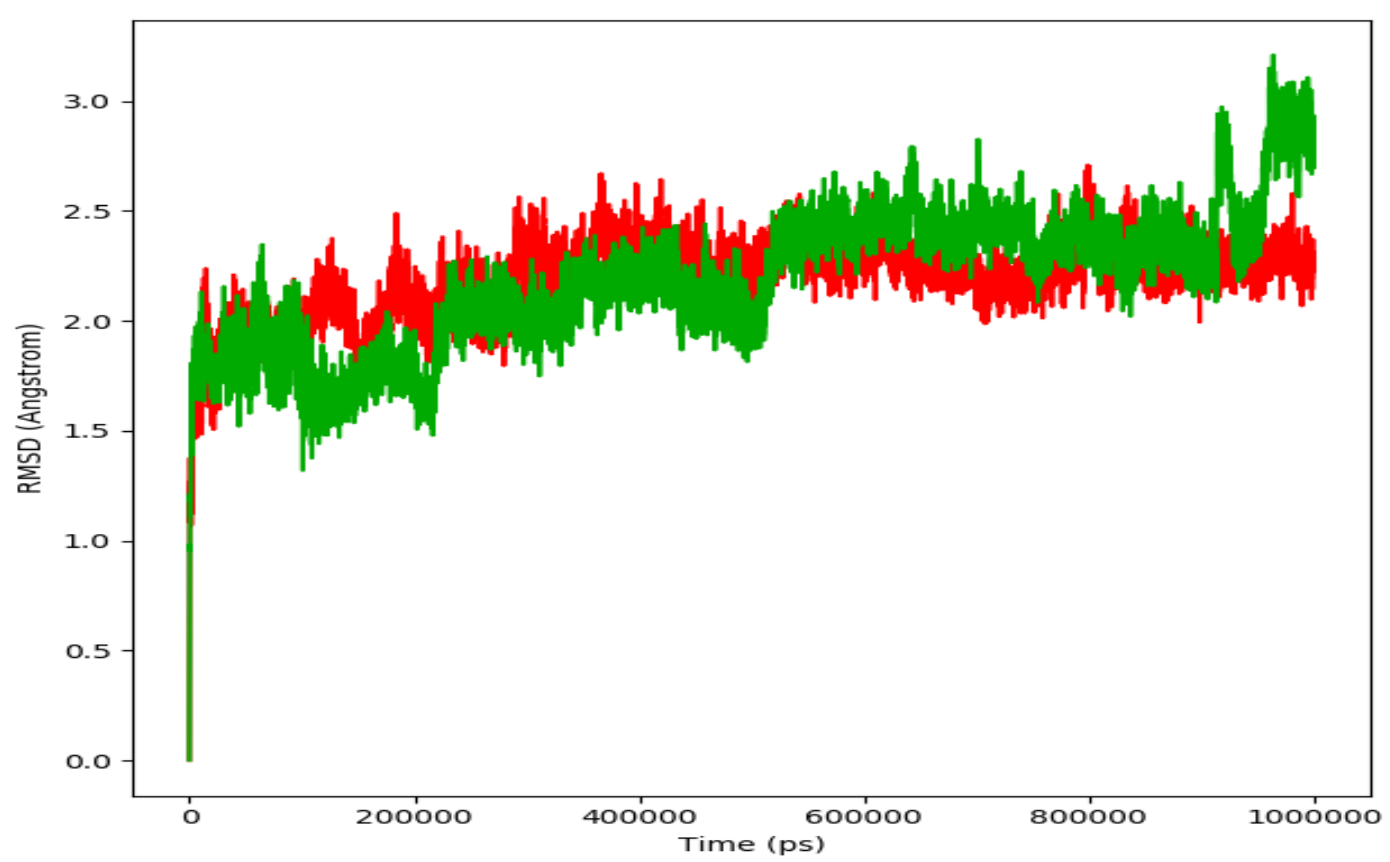

Figure 2: RMSD analysis graph of the inactive (red) at $\mathrm{pH} 7.5$ and active (green) at $\mathrm{pH} 8.4 \mathrm{CA}-\mathrm{II}$ protein. 

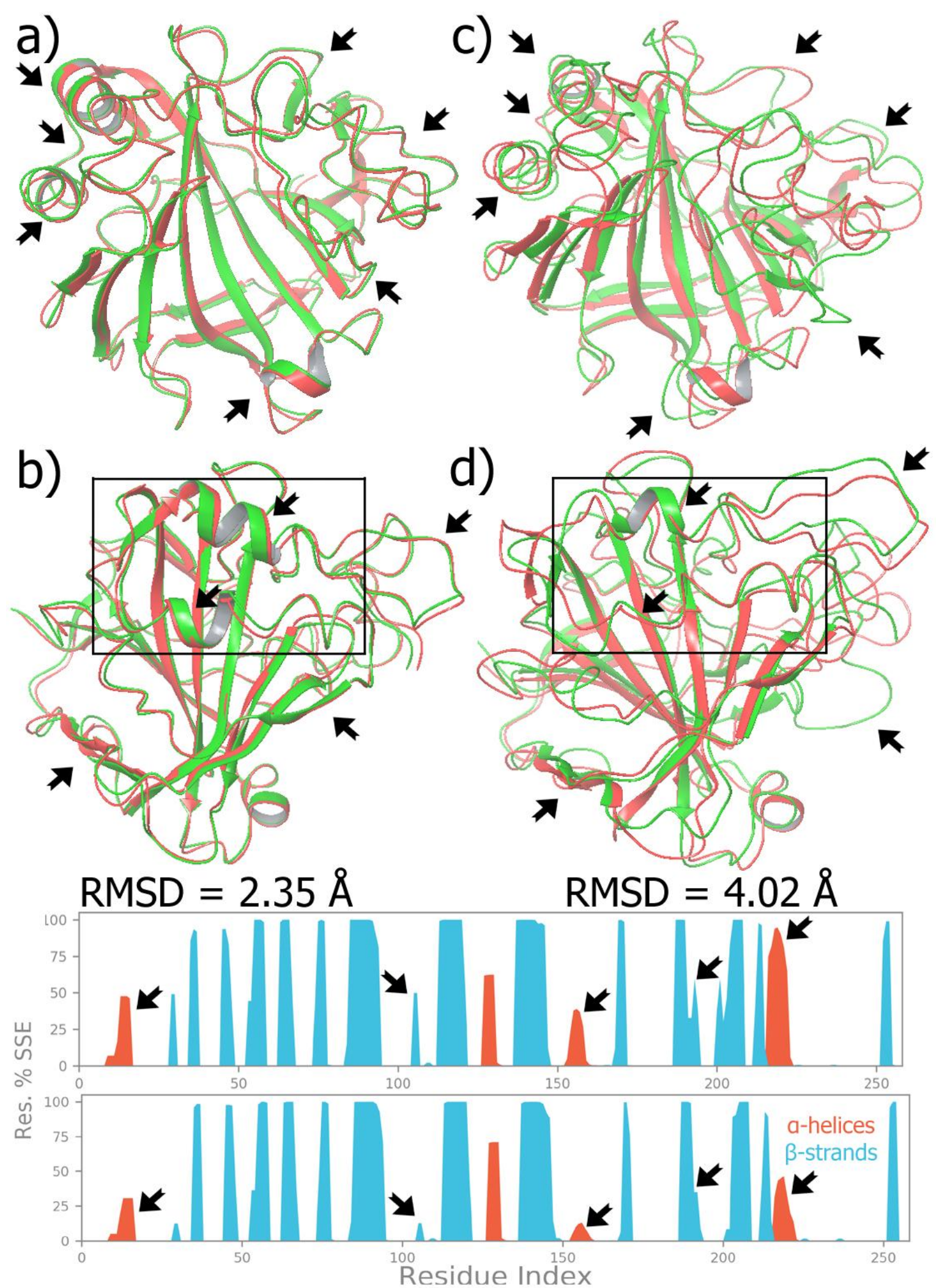

Figure 3: Superimposed view of the inactive (red) at $\mathrm{pH} 7.5$ and active (green) at $\mathrm{pH} 8.4$ CA-II protein sampled from the a,b) first frame and $\mathbf{c , d}$ ) last frame of the simulated time scale, depicting the loss of some of the key secondary structural elements such as alpha-helices and beta-strands observed during the simulation. 
Notable conformational changes in the CA-II were observed between first (figure 3a,b) and last frames (figure $3 \mathrm{c}, \mathrm{d}$ ) superimposition sampled from respective simulations of CA-II at $\mathrm{pH} 7.5$ and at $\mathrm{pH}$ 8.4. Rise in RMSD difference from 2.35 to $4.02 \AA$ are observed to be due to the conformational changes occurred in CA-II at $\mathrm{pH} 8.4$, especially at $100^{\text {th }} \mathrm{ns}, 200^{\text {th }} \mathrm{ns}, 500^{\text {th }} \mathrm{ns}$ and $900^{\text {th }}$ ns as evident from figure 1. While, there was intermittent jumps observed in backbone RMSD of CA-II, However, interestingly no considerable changes were observed in total number of intra molecular hydrogen bonds observed during the entire simulated timescale (figure 4).

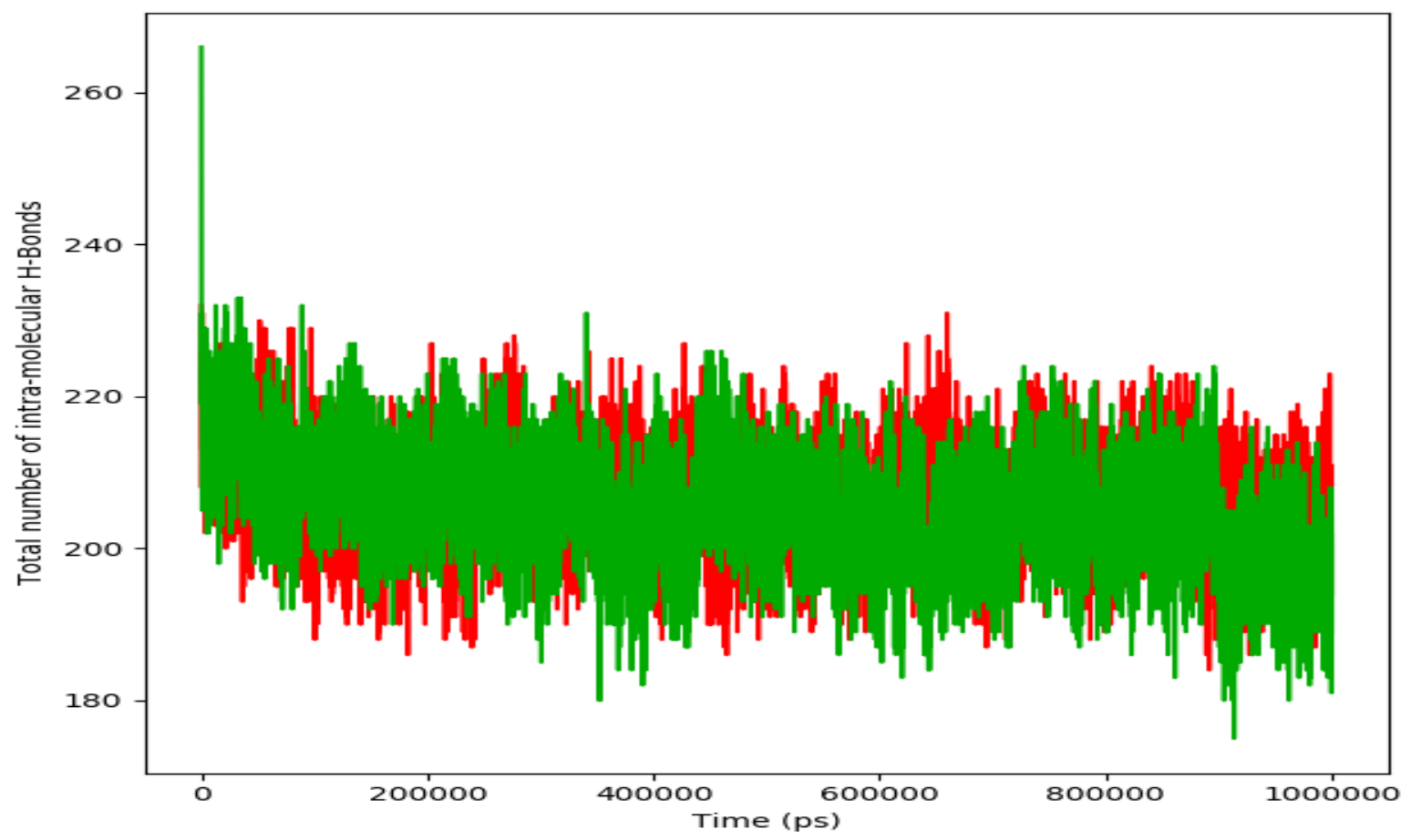

Figure 4: Intra molecular hydrogen bonds analysis graphs of the inactive (red) at $\mathrm{pH} 7.5$ and active (green) at $\mathrm{pH}$ 8.4 CA-II protein.

On the other hand, significant jump in the activity of individual residues RMSF up to $6 \AA$ was observed. Major fluctuations in the residues Phe20 to Pro30; Gln53; Asp72, Asp75; Lys80; Glu106; His107; Glu117; Thr125 to Gly145; Val150 to Lys170 and Thr199 were observed when simulations of CA-II at $\mathrm{pH} 7.5$ and at $\mathrm{pH} 8.4$ were analyzed as shown in figure 5. Among the observed fluctuations, significant difference was noted for residues Phe20 to Pro30 and Val150 to Lys170. Since, it is common to observe that unattached $C \& N$ terminals of the proteins fluctuates high in the simulated environment, we focused on Val150 to Lys170 residues to investigate further based on their importance in forming CA-II dimers and tetramers. As pointed out with small arrows in figure 6, dramatic conformational changes have been observed for residues between Val150 to Lys170, especially Val150, Gly151, Lys154, Lys159 and Ser166. These observed changes can been seen reflected in figure $3 b \& d$, where the alpha-helix involving residues between Val150 to Lys170 was observed to the losing its Secondary Structural Elements (SSE) thus its structural integrity (figure 3). 


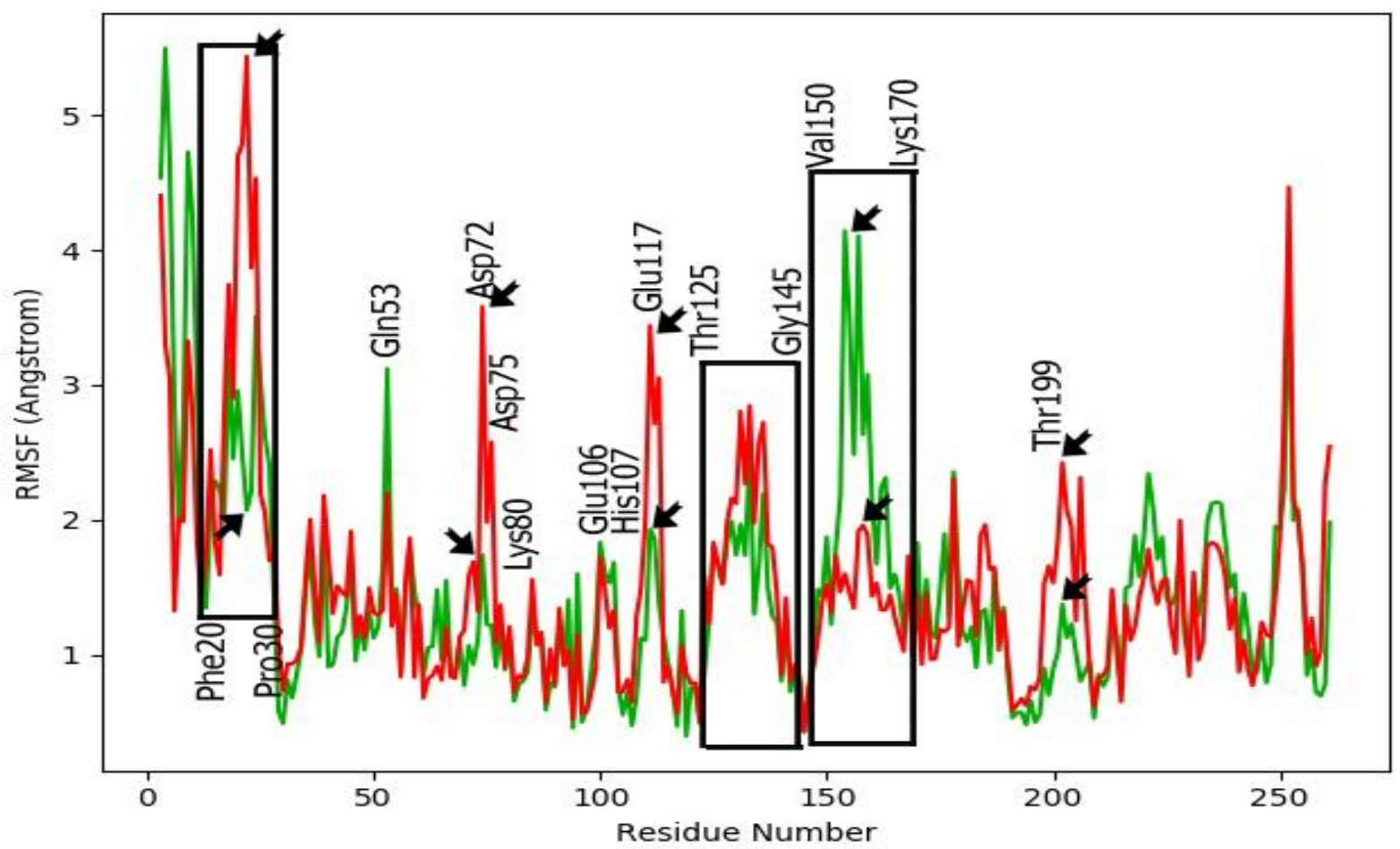

Figure 5: RMSF analysis graph of the inactive (red) at $\mathrm{pH} 7.5$ and active (green) at $\mathrm{pH} 8.4 \mathrm{CA}$-II protein showing some of the residues involved in fluctuations and structural changes observed during simulated timescale.
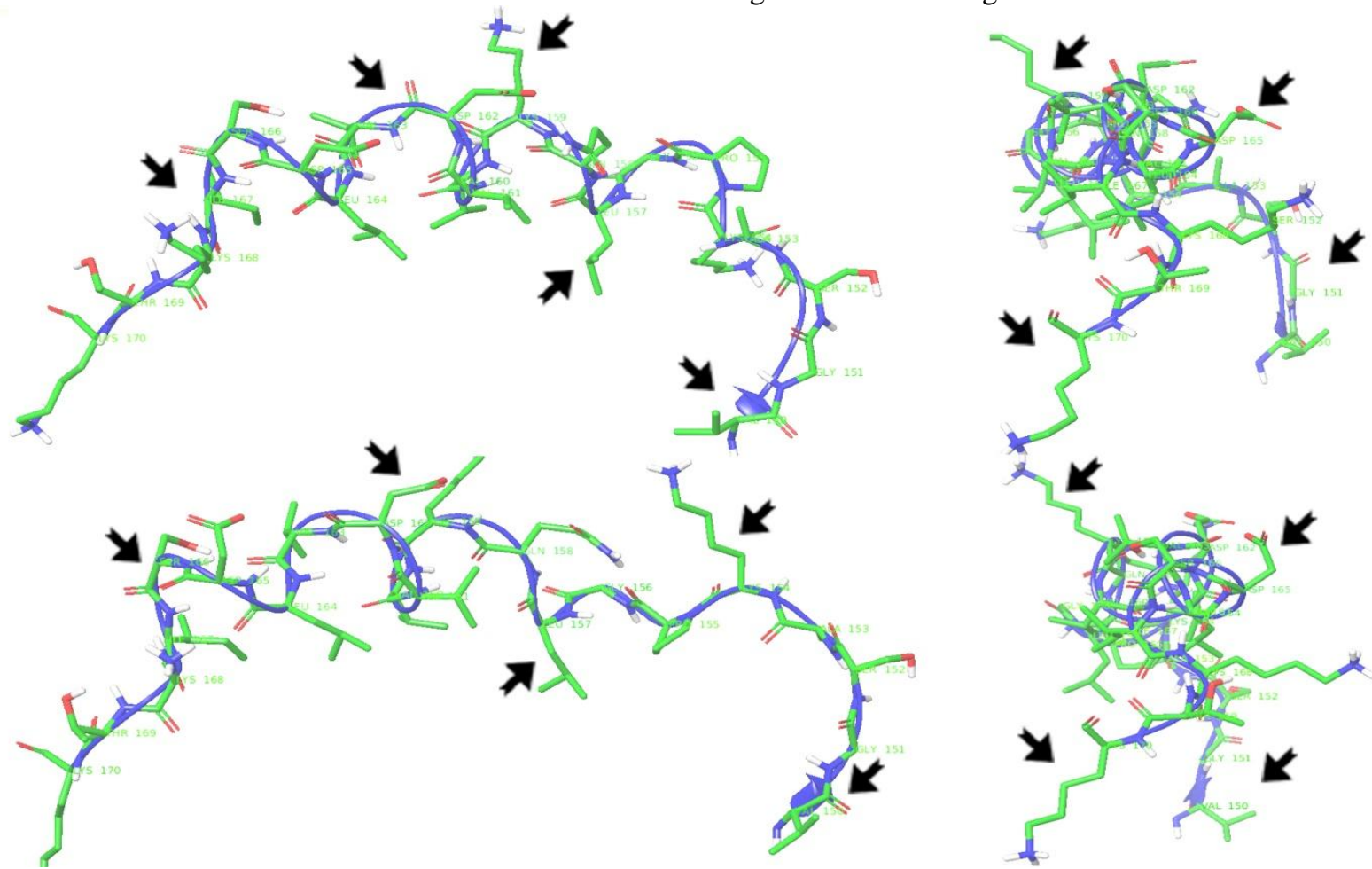

Figure 6: Front view (left) and side view (right) of the residues between 150-170 amino acid numbers which are involved in some of the high fluctuations observed during simulated timescale. Small arrows indicate the key movements observed for the residues. Above panels indicate first frame of the simulation and below panels indicate last frame of the simulated time scale. 


\section{Analysis of the gatekeeper residues Glu106 and Thr199:}

Keeping in view of the importance of Glu106 and Thr199 as gatekeeper residues reported in earlier studies, we have analyzed the conformational changes in the protein around these residues. As observed in RMSF plot (figure 5), Thr199 have shown a major fluctuations from its position, which was thought to be triggered the other conformation changes observed in CA-II (figure 3), including the loss of SSE percentage around 150-170 residues. To further understand, what exactly happening around these gatekeeper residues, we have analyzed the distance between $\mathrm{Zn}$ ion, Glu106 \& Thr199. As shown in figure 7, distinct difference between the active and inactive forms of CA-II residual positions has been noted.

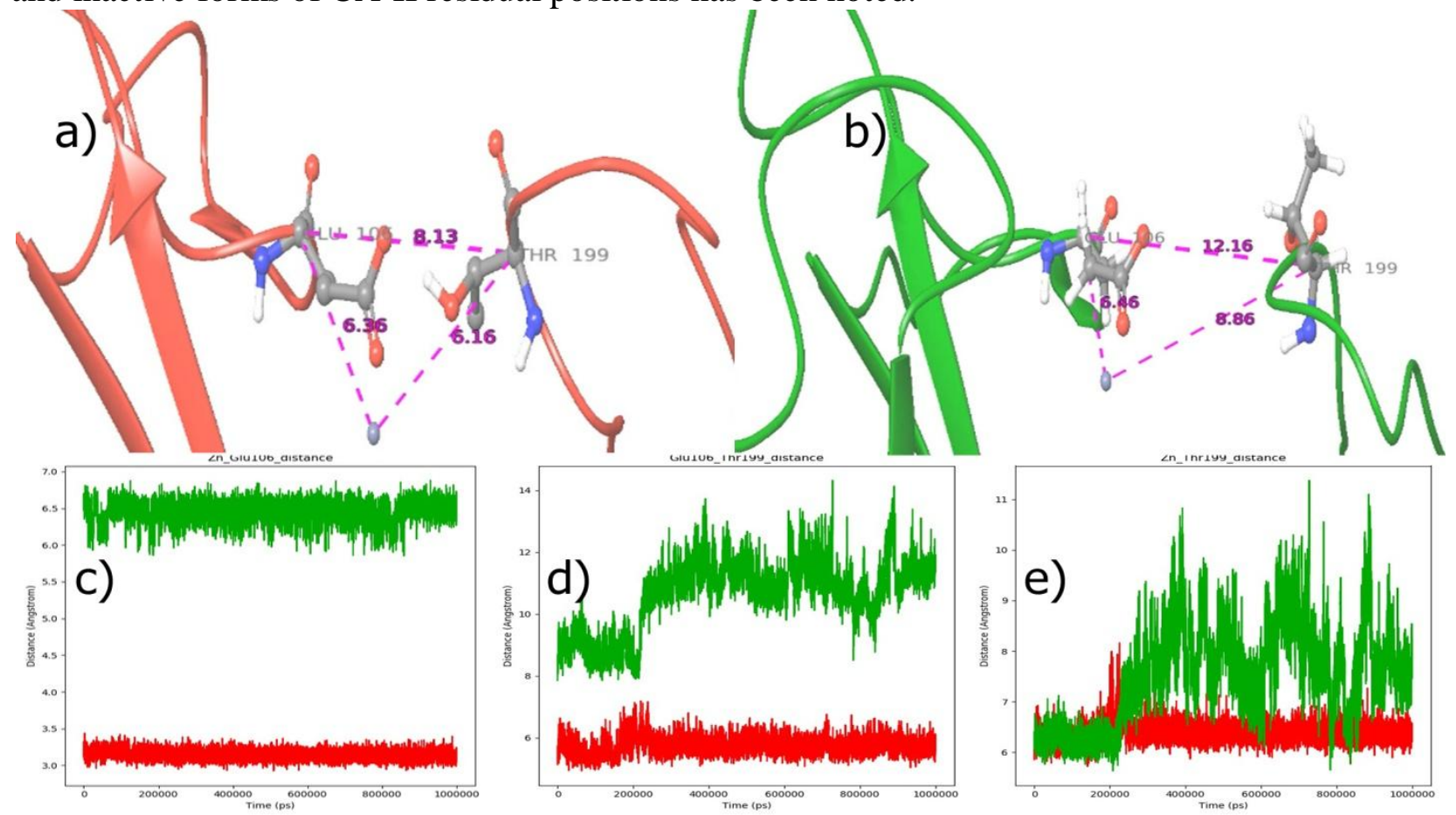

Figure 7: Represents the distances between Zn, Glu106 \& Thr199 residues from the randomly sampled snapshot from the simulated timescale of MD simulations. a) in red color ribbons and loops represents the snapshot from simulation of CA-II at $\mathrm{pH} 7.5$ (inactive) and b) in green color ribbons and loops represents the snapshot from simulation of CA-II at $\mathrm{pH} 8.4$ (active) c) Depicts the calculated distance between $\mathrm{Zn}$ ion and Glu106 residue d) Depicts the calculated distance between Glu106 and Thr199 residues e) Depicts the calculated distance between $\mathrm{Zn}$ ion and Thr199 residue. Red color graphs represent the simulation of CA-II at pH 7.5 (inactive) and Green color graphs represent the $\mathrm{CA}-\mathrm{II}$ at $\mathrm{pH} 8.4$ (active).

When the distance between the $\mathrm{Zn}$ ion \& Glu106 (figure 7c), Zn ion \& Thr199 (figure 7d) and Glu106 \& Thr199 (figure 7e) was analyzed, it was revealed that the residual position in case of inactive form for these gatekeeper residues were quite rigid and stabilized in nature compared to its active form. As evident from the figure 7d,e; a sudden flip of Thr199 residue at around 200ns of simulated timescale has resulted in sudden rise in the distance with Glu106 and $\mathrm{Zn}$ ion. This event of Thr199 sudden flip is what believed to be the key incident which causes the entry / restriction of the substrate at the catalytically active site coordinated by $\mathrm{Zn}$ ion via converting the $\beta$-sheet to loop, thus providing the space required for the substrate entry. From our simulation analysis, it was visualized that the loop which co-ordinates the Thr199 residue acts as a lid in the substrate entry path as shown in figure 8 . Some of the randomly sampled simulation snapshots 
depicting the conformational changes in the "lid-like acting loop" in active and inactive forms of CA-II are shown in figures $9 \& 10$.

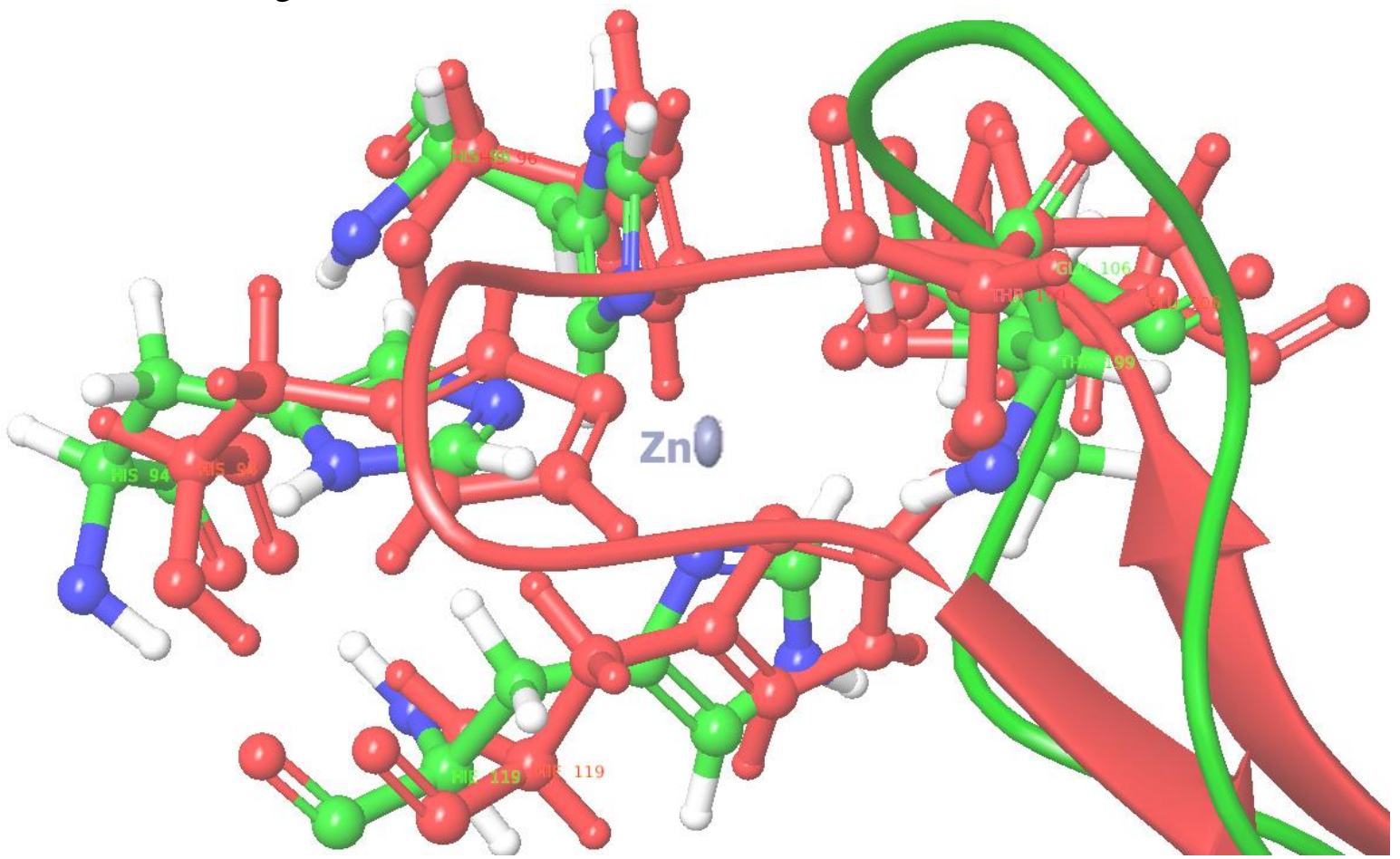

Figure 8: Superimposed view of the inactive (red) at $\mathrm{pH} 7.5$ and active (green) at pH 8.4 CA-II protein sampled from the last frame of the simulated time scale, depicting key gatekeeper residues Glu106, Thr199 fluctuations along with the loss of beta-strands in its inactive state (red) to loops in active state (green) observed during the simulation.

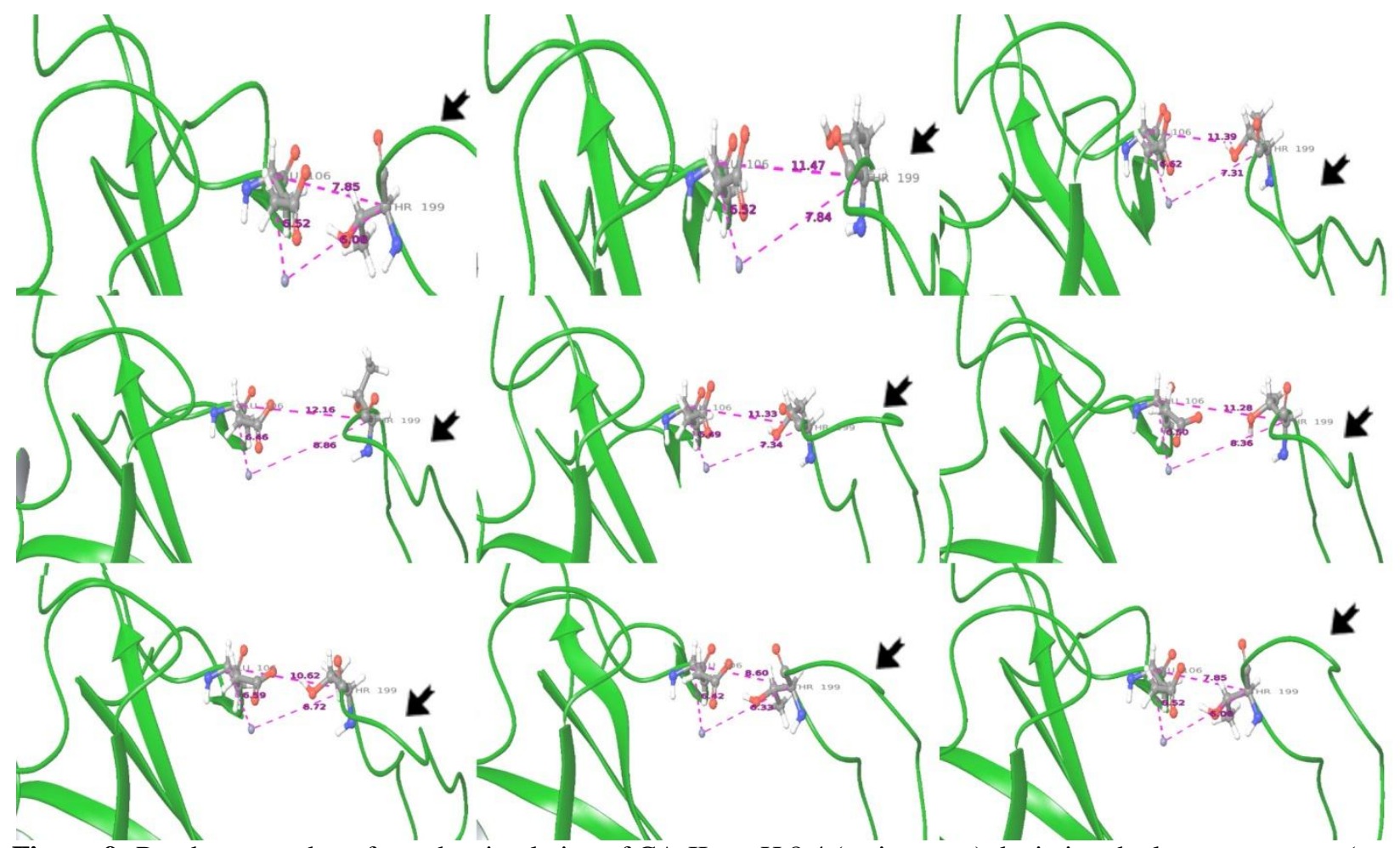

Figure 9: Random snapshots from the simulation of CA-II at pH 8.4 (active state) depicting the loop movement (see small arrows) coordinated by Thr199 residue responsible for its activity. 


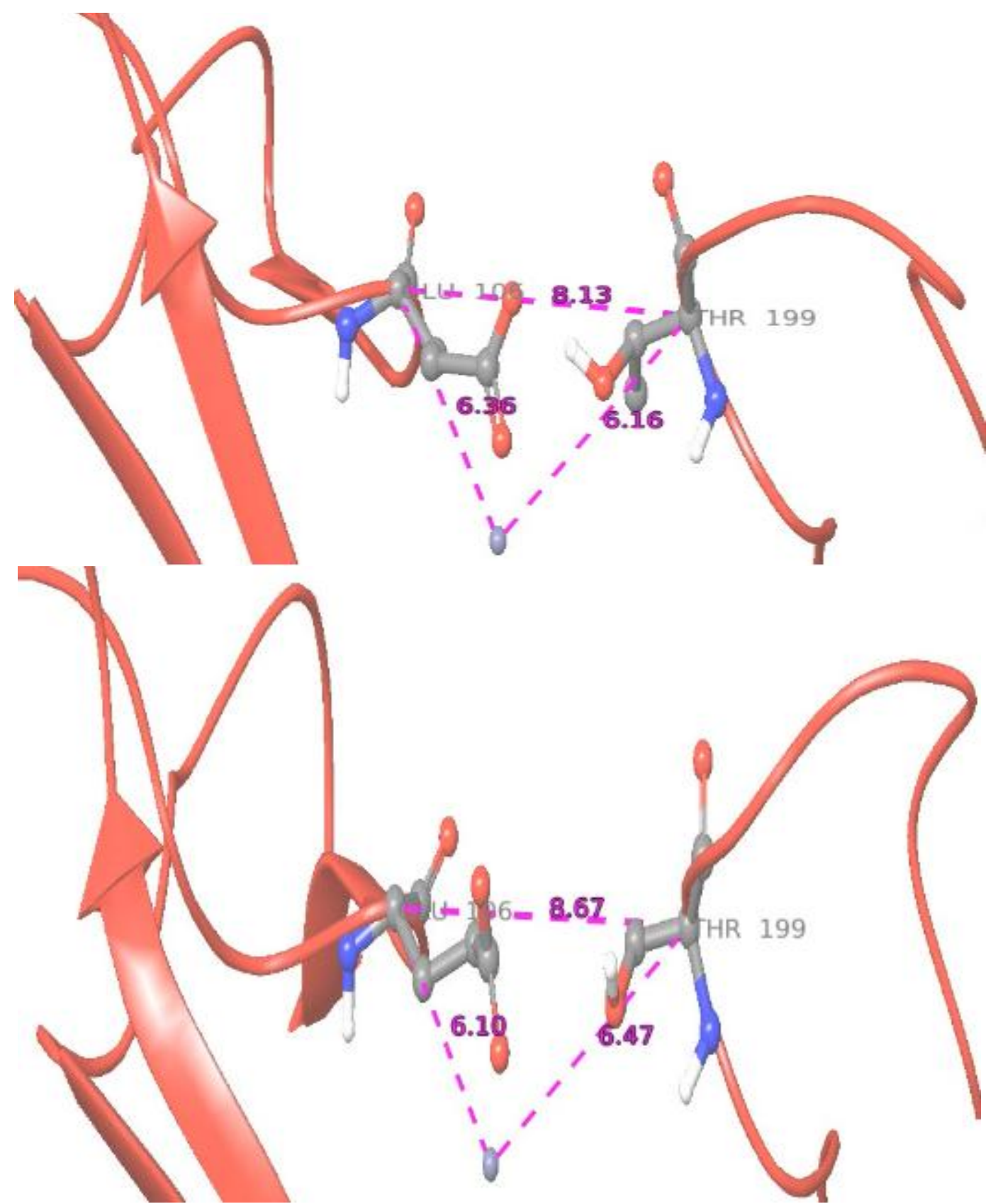

Figure 10: Random snapshots from the simulation of CA-II at pH 7.5 (inactive state) depicting the loop movement coordinated by Thr199 residue responsible for its activity.

\section{Conclusion:}

In this present study, we have employed long time scale microsecond level all atomic molecular dynamic simulation to understand the dynamics of CA-II in its active and inactive forms. From our analysis it was hypothesized that sudden flip of the Thr199 residue triggers a series of conformational changes in the CA-II including loss of some critically important secondary structural elements, especially with loop coordinated by Thr199 and alpha-helix formed by Val150 to Lys170 residues responsible for substrate entry and dimers / tetramers formation 
respectively. The present knowledge will be of high value when designing strategies on design / discovery novel CA-II activators or inhibitors.

Authors' contribution: Pulala Raghuveer Yadav designed the study. Syed Hussain Basha and Pulala Raghuveer Yadav executed the work and generated the data. Pulala Raghuveer Yadav, Syed Hussain Basha and Pavan Kumar Pindi analyzed the data and wrote the manuscript. All authors have read and approved the final manuscript.

Conflicts of Interest: The authors declare that they have no conflict of interests.

\section{References:}

1. Zhang, Xiaoping, et al. "Kinetics and mechanism of the hydration of carbon dioxide and dehydration of bicarbonate catalyzed by a zinc (II) complex of 1, 5, 9triazacyclododecane as a model for carbonic anhydrase." Inorganic Chemistry 32.25 (1993): 5749-5755.

2. Graham, D., and M. L. Reed. "Carbonic anhydrase and the regulation of photosynthesis." Nature New Biology 231.20 (1971): 81-83.

3. Supuran, Claudiu T. "Structure and function of carbonic anhydrases." Biochemical Journal 473.14 (2016): 2023-2032.

4. Eriksson, A. Elisabeth, T. Alwyn Jones, and Anders Liljas. "Refined structure of human carbonic anhydrase II at $2.0 \AA$ resolution." Proteins: Structure, Function, and Bioinformatics 4.4 (1988): 274-282.

5. Kanth, Bashistha Kumar, Jinwon Lee, and Seung Pil Pack. "Carbonic anhydrase: Its biocatalytic mechanisms and functional properties for efficient $\mathrm{CO} 2$ capture process development." Engineering in Life Sciences 13.5 (2013): 422-431.

6. Yadav, Raju R., et al. "Carbonic anhydrase mediated carbon dioxide sequestration: Promises, challenges and future prospects." Journal of basic microbiology 54.6 (2014): 472-481.

7. Kanth, Bashistha Kumar, Jinwon Lee, and Seung Pil Pack. "Carbonic anhydrase: Its biocatalytic mechanisms and functional properties for efficient $\mathrm{CO} 2$ capture process development." Engineering in Life Sciences 13.5 (2013): 422-431.

8. Lindskog, Sven, and David N. Silverman. "The catalytic mechanism of mammalian carbonic anhydrases." The carbonic anhydrases. Birkhäuser, Basel, 2000. 175-195.

9. Schrödinger Release 2013-2: Maestro, Schrödinger, LLC, New York, NY, 2013.

10. Sussman, Joel L., et al. "Protein Data Bank (PDB): database of three-dimensional structural information of biological macromolecules." Acta Crystallographica Section D: Biological Crystallography 54.6 (1998): 1078-1084.

11. Schrödinger Release 2020-2: Desmond Molecular Dynamics System, D. E. Shaw Research, New York, NY, 2020. Maestro-Desmond Interoperability Tools, Schrödinger, New York, NY, 2020.

12. McDonald, Nora A., and William L. Jorgensen. "Development of an all-atom force field for heterocycles. Properties of liquid pyrrole, furan, diazoles, and oxazoles." The Journal of Physical Chemistry B 102.41 (1998): 8049-8059. 
13. Mark, Pekka, and Lennart Nilsson. "Structure and dynamics of the TIP3P, SPC, and SPC/E water models at 298 K." The Journal of Physical Chemistry A 105.43 (2001): 9954-9960.

14. Covarrubias, Adrian Suarez, et al. "Structural mechanics of the $\mathrm{pH}$-dependent activity of $\beta$-carbonic anhydrase from Mycobacterium tuberculosis." Journal of Biological Chemistry 281.8 (2006): 4993-4999. 